\title{
LA INTRODUCCIÓN DE LA PRÁCTICA QUÍMICA EN LA REAL BOTICA ESPAÑOLA*
}

\author{
Pilar García de Yébenes Torres
}

Doctora en Farmacia.

\section{M. ${ }^{\mathrm{a}}$ Luisa de Andrés Turrión}

Doctora en Farmacia. Profesora titular del Departamento de Farmacia y

Tecnología Farmacéutica, de la Facultad de Farmacia, U.C.M.

\section{RESUMEN}

Los intentos de introducir la aplicación de la química en la elaboración de medicamentos en la Real Botica española se harán realidad definitivamente, coincidiendo con la llegada a la corte de Felipe V y de su equipo de sanitarios: médicos, cirujanos y boticarios.

\section{SUMMARY}

The introduction of Chemist in the elaboration of medicines in the Spanish Royal Pharmacy will finally be done effective at the same time that the proclamation of King Philipe V.

Analizar el momento en que las primeras prácticas químico-farmacéuticas tuvieron lugar en la Real Botica, organismo creado y protegido por la monarquía española de finales del siglo XVI y absolutamente supeditado a los criterios de sus rectores, los médicos de Cámara de S.M., supone recapacitar sobre cuál fue el reconocimiento sanitario oficial, es decir, del Real Tribunal del Protomedicato, al llamado sistema iatroquímico, a la aplicación de la farmacoquímica y cuál la actitud del colectivo profesional de boticarios españoles.

* Financiado con cargo al proyecto de investigación PS94-0028 del Programa Sectorial de Promoción General del Conocimiento. 


\section{PILAR GARCÍA DE YÉBENES TORRES Y M. ${ }^{a}$ LUISA DE ANDRÉS TURRIÓN}

\section{ANTECEDENTES.}

La difusión de la obra de Paracelso y el nacimiento de la iatroquímica tuvieron como consecuencia la introducción de la práctica química en la actividad farmacéutica a lo largo de los siglos XVI y XVII. El movimiento iatroquímico y sus aspiraciones de alcanzar un sistema médico moderno integrado por todo tipo de novedades, encontró la oposición de los galenistas, aferrados aún a las doctrinas tradicionales. Este enfrentamiento, durante dos o tres décadas, finalizará antes de terminar el siglo XVII. La influencia de Boyle al constituir la química moderna, el progreso de las investigaciones anatomofisiológicas, así como las bases implantadas por Sydenham en su método nosológico, prescindiendo de hipótesis fisiológicas, acabaron por derrumbar el sistema iatroquímico.

En España, este proceso fue más lento. Las ideas de Paracelso no obtuvieron prácticamente ninguna divulgación en el siglo XVI. Pese a todo, en los últimos años de esta centuria, diferentes profesionales trabajaron al servicio del monarca Felipe II con gran interés por relacionar la química y la medicina ${ }^{1}$. Será precisamente Felipe II quien, en 1594, funde una institución que garantizara el servicio de medicinas a toda la Casa Real, a la que denominaría Real Botica y a la que pondría bajo la responsabilidad de un boticario mayor.

El boticario mayor, en aquellos momentos, era un individuo experto en el arte de la preparación de medicamentos, cuya misión primordial consistía en elaborar aquéllos que los médicos, bien de Cámara (que recetaban exclusivamente al rey y a su familia) o bien de familia (que atendían a todos los criados reales) le ordenaran. Su trabajo estaba controlado muy de cerca por los primeros que, al mismo tiempo, integraban el Real Tribunal del Protomedicato, y por los jefes de la Casa y Cámara Real, mayordomo mayor y sumiller de corps respectivamente. El arsenal de medicamentos que allí se preparaban era mayoritariamente tradicional, aunque se detectaron nuevas aplicaciones de la química, sobre todo en la obtención de "quintaesencias" por destilación.

Durante el reinado de Felipe IV, el servicio de la Farmacia Real inició importantes cambios frente a etapas anteriores. Diego de Cortavila Sanabria, boticario mayor (1641-1649)2 , que pasó toda su vida profesional en la Casa Real consiguiendo el

1 En 1597, Diego de Santiago, quien trabajó en el laboratorio de Destilación de El Escorial, vió publicada su obra "Arte Separatoria", considerada por López Piñero como el mejor texto químico del siglo XVI en España. Lorenzo Cózar fue un defensor de Paracelso, de sus ideas y teorías, llegando a impartir en Valencia enseñanzas químicas con la creación de una cátedra de química en esta ciudad. FOLCH JOU, G., SUÑÉ ARBUSSÁ, J.M., VALVERDE LóPEZ, J.L. (1986) Historia General de la Farmacia, el medicamento a través del tiempo, vol. 1, $1^{\text {a }}$ edición, Madrid, Ediciones Sol S.A., p. 279.

2 VAlVERDE, J.L., S. TÉLLEZ, M.C. (1976), "Diego de Cortavila y Sanabria, Boticario Real (15701650)". Boletín de la Sociedad Española de Historia de la Farmacia (B.S.E.H.F.), 105, pp. 3-15. 


\section{LA INTRODUCCIÓN DE LA PRÁCTICA QUÍMICA EN LA REAL BOTICA ESPAÑOLA}

agradecimiento y consideración científica de sus rectores sanitarios, junto con su equipo de colaboradores, logró que se elaboraran normativas para el control del funcionamiento de la Real Botica (las primeras, y hasta entonces últimas, instrucciones fueron redactadas en 1594): unas reglamentaciones de detalle, en 1645, y una extensa Etiqueta, en 1649, que se mantendrá en vigor más de un siglo. En esta última de 18 de abril de 1649, por primera vez, se controlaba la elaboración y el despacho de preparados químicos 3 :

\footnotetext{
“...11. Que no se pueda dar ninguna medicina química si no fuere por receta de los Médicos de Cámara."

“...19. El preparar las perlas... y todas las demás cosas que se preparan... y todas las demás medicinas compuestas de cualquier género que sean, se haga todo en la Botica de S.M. por los oficiales de ella, pues hay bastantes, sin que se pueda comprar ningún medicamento compuesto hecho fuera de la Botica."
}

El margen de confianza que la jerarquía sanitaria palaciega otorgó a los boticarios reales en aquel momento fue muy importante. Pese a la rigidez y dureza con que la terapéutica química había sido recibida por los médicos de Cámara de Felipe IV (su Real Protomedicato), la elaboración de medicamentos químicos fue admitida en la Real Botica. Nunca hasta entonces las relaciones entre el personal de medicina y farmacia de la Real Cámara habían sido más cordiales; probablemente, el buen hacer de los boticarios reales y/o su proximidad a las más altas instancias sanitarias hicieron reflexionar al monarca español sobre la profesión farmacéutica declarándola, pocos meses después (1650) "arte científico"4. La consideración social de los boticarios no cambió tras la promulgación de esta disposición real, ni siquiera el método de aprendizaje profesional que se mantendría como antaño: maestro-ayudante.

FOLCH JOU, G. (1964), "El título de visitador de Boticas de Diego Cortavila y Sanabria, consecuencias que del mismo se desprenden". B.S.E.H.F., 59, pp. 99-107.

3 De los puntos concernientes a la Real Botica de la Etiqueta de 18 de abril de 1649 para el gobierno de la Real Cámara. ARCHIVO GENERAL DEL PALACIO REAL DE MADRID (A.G.P.), Sección Histórica, caja 54. En GARCÍA DE YÉBENES TORRES, P. (1994) La Real Botica durante el reinado de Felipe V (17001746), Tesis Doctoral inédita, D.F. y T.F., Facultad de Farmacia, U.C.M., Madrid, 373-382.

4 FOLCH ANDREU, R. (1927) Elementos de Historia de la Farmacia, Madrid.

FOLCH JOU, G. (1972) Historia de la Farmacia, $3^{\mathrm{a}}$ edición, Madrid, p.244.

Gómez CAAMAÑo, J.L. (1982) Páginas de Historia de la Farmacia, Barcelona. 


\section{PILAR GARCÍA DE YÉBENES TORRES Y M. ${ }^{a}$ LUISA DE ANDRÉS TURRIÓN}

\section{El REAL OFICIO DE LA QUímiCA (1694-1723).}

En 1694, el Real Tribunal del Protomedicato autorizó el nacimiento de un organismo independiente, como ya lo fuera la Real Botica de S.M., integrado en la Real Cámara de la Casa Real española. Se denominaría Real Oficio de la Química, aunque vino a llamarse Real Laboratorio de la Química5. Carlos II, aconsejado por alguno de sus asesores sanitarios más próximos, decidió “...separar de su botica galénica, el arte chímica" creando las plazas de spargírico mayor (o manipulante chimico) con sus ayudas subalternos ${ }^{6}$.

Todo hacía indicar que aquellos interrogantes que Juan de Cabriada expusiera en su Carta filosófica, médico-chymica (1687), calificada actualmente como el principal manifiesto del movimiento renovador de la medicina española, habían conseguido respuesta afirmativa:

\footnotetext{
“....¿Por qué, no se adelantará y se promoverá este género de estudio? ¿Por qué, para poderlo conseguir, no se fundará en la Corte del Rey de España una Academia Real, como la hay en la del Rey de Francia, en la del de Inglaterra y en la del señor Emperador? ¿Por qué para un fin tan santo, útil y provechoso, como adelantar en el conocimiento de las cosas naturales (sólo se adelanta con los experimentos físico-químicos) no habían de hincar el hombro los señores y nobleza, pues esto no les importa a todos menos que las vidas? ¿Y por qué en una Corte como ésta, no había de haber ya una oficina química, con los más peritos artífices de Europa? Pues la Majestad Católica de Rey nuestro señor; que Dios guarde, los tiene en sus dilatados reinos, de donde se podrían traer los mejores."
}

Los preliminares a la fundación del Real Laboratorio de la Química supusieron un debate cuyo desenlace fue favorable al empleo de los remedios químicos. Los ambientes científicos a favor o en contra de la Química hervían en la última década del siglo XVII. También había variado la opinión del Protomedicato ya que pocos

\footnotetext{
5 Este tema aparece analizado en:

VEGA PORTILlA, J., (1946) La Botica Real durante la dinastía austriaca, Tesis Doctoral, Facultad de Farmacia U.C.M., Madrid pp. 349-406.

VALVERDE, J.L.; SÁNCHEZ TÉLlez, C., (1977) "El laboratorio químico de la Real Botica (16931700)". Ars Farmacéutica, 18, pp. 121-152.

GAGO, R.; OlAGÜE, G.; CARRILlO, J.L., (1981) “Aportación al estudio del movimiento novator en España. El Laboratorio Químico del Palacio Real (1694), la obra del boticario aragonés Juan del Bayle (1698)". (B.S.E.H.F.), 126, pp. 95-107.

6 A mediados del siglo XVIII surgió un grave enfrentamiento en la Real Botica entre dos de sus boticarios: el boticario mayor Bartolomé Pérez Durán y el ayuda más antiguo de espargírico Francisco Corral. En las alegaciones e informes como consecuencia del enfrentamiento se informó de la antigüedad de las prácticas químicas y su incorporación en aquella oficina. A.G.P., Sección Administrativa, leg. 625, Empleos de la Real Casa, Ayudas de la Botica.
} 


\section{LA INTRODUCCIÓN DE LA PRÁCTICA QUÍMICA EN LA REAL BOTICA ESPAÑOLA}

meses antes de la fundación del Real Laboratorio de la Química, Juan de Cabriada, entusiasta de la Química, intentó fundar una Academia Espargírica en Madrid; en el año 1693, en unión del cirujano real del Buen Retiro, Cristobal de León, enviaban un memorial al Rey proponiendo tal creación. Pero, pasado al Tribunal del Protomedicato, no creyeron conveniente difundir la doctrina química. Esta idea estaba denostada por el Duque de Frías, Iñigo Melchor Fernández de Velasco y Tovar, mayordomo mayor, y dió con todo al traste.

\section{2.a. Vito Cataldo, espargírico mayor de S.M. (1694-1698).}

El 9 de agosto de 1694, el médico de Cámara, Dionisio Cardona, uno de los prologuistas de aquel texto de Cabriada, presentó un informe titulado Sobre la química ${ }^{7}$ en el que, entendiendo ya habitual el empleo de medicamentos químicos, instaba al Protomedicato para que en sus visitas de inspección a las boticas del reino exigieran a sus boticarios propietarios la tenencia de estos productos para su despacho, obligándoles, para evitar que estuvieran mal preparados, a adquirirlos ya elaborados en el Real Laboratorio. La respuesta de dicho Tribunal fue positiva ${ }^{8}$. La Casa Real, representada por el sumiller de corps, así como el pleno de los médicos de Cámara y de boticarios reales, autorizaron la creación del Oficio de la Química y determinaron la suficiencia del napolitano Vito Cataldo como "manipulante de la medicina química" o "espargírico mayor"; éste, y sus dos empleados, Juan Bautista Pizzi y Nicolás Crezenzo, napolitanos también, quedaban conectados con la Real Botica pretendiendo trabajar con absoluta autonomía jerárquica y económica9. Dos médicos de Cámara, Andrés Gámez y el ya citado Cardona, fueron nombrados directores de las mani-

7 Informe amplio. A.G.P., Sección Administrativa, leg. 429.

López Piñero ha estudiado a fondo la figura de Cabriada. Entre los trabajos a él dedicados resaltamos IPEZ PIÑERO, J.Ma . (1965) "La Carta filosófica, médico chymica (1687) de Juan de Cabriada, punto de 'ida de la Medicina moderna en España", Asclepio, XVII, pp. 207-214.

8 A.G.P., Libro de Registro $n^{\circ} 557$.

Cuando Vito Cataldo llega a España, para servir en la Corte como químico, a comienzos del año e especuló con la conveniencia de que se le hiciera asiento (de personal) en la Real Botica de

no sólo a él, también a sus dos ayudantes. En este sentido, se pedía al sumiller de corps informara s.M.; no tenemos constancia del contenido del informe, aunque sí de su existencia. A.G.P., Libro de Registro $n^{\circ}$ 557. Folio 54/32 vto. y 55/33.

Juraron el 29 de enero de 1694. Se les asignaron, a Cataldo 15 Reales de Vellón (Rv.)/día, y a los ayudas 7 Rv. 17 Maravedíes de Vellón (Mrv.) a cada uno, a pagar por la consignación de la Real Casa, desde 24 de septiembre de 1693, que salieron de Nápoles. Según certificación de J.M. de Zeaorrote. A.G.P., Expedientes personales, número 2613/18. 


\section{PILAR GARCÍA DE YÉBENES TORRES Y M. ${ }^{a}$ LUISA DE ANDRÉS TURRIÓN}

pulaciones en los remedios químicos con la misión de controlar tanto las peticiones como la elaboración de los mismos ${ }^{10}$.

Uno de los aspectos que más preocupaban a la Real Hacienda era la administración de este nuevo estamento. Se acordó que el espargírico mayor debería presentar cuentas separadas de las del Oficio de la Botica. El abastecimiento de los productos y materiales del Laboratorio se realizaría desde alguna droguería de la calle, mediante cédula de un contador, presentándose después las facturas a la Veeduría y Contaduría de la Real Cámara ${ }^{11}$. Todos los oficiales tendrían sus sueldos y consignaciones separados de los de la Botica, sin tener que realizar ningún tipo de asistencia en ella; su única obligación sería la elaboración y despacho de los medicamentos químicos que recetaran los médicos de Cámara.

Vito Cataldo presentó una relación de las preparaciones químicas que se podían comenzar a elaborar en el Real Laboratorio de la Química' ${ }^{12}$. En dicha relación aparecen agrupados los remedios según su origen, es decir: "de los vegetales, de la cera, de la miel, del maná, de la canela, del espíritu de vino y otros menstruos, del tártaro, del coral y otras piedras preciosas, de los metales del oro y de la plata, del acero, del cobre, del plomo, del estaño,... de los animales"13.

Superando las 150 elaboraciones (151 mencionadas expresamente, si bien algunas pueden contener muchas más al ser grupos de preparados, por ejemplo "Tinturas con balsamos diversos"), componen un repertorio terapéutico amplio y tan completo - en ningún caso encaminado a solucionar un solo tipo de patologías- que, de haber funcionado el Oficio de la Química con apoyo oficial decidido y firme, hubiera llegado a ser un serio competidor de la Real Botica, hegemónico organismo en la elaboración de medicamentos para la Casa Reạl.

Aparecen reseñados todo tipo de aceites, aceros, elixires, espíritus, extractos, tinturas, sales, etc, esencialmente derivados de minerales (plomo, antimonio, cobre,

10 Según un informe del sumiller de corps, registrado con fecha 18 de abril de 1694, había doctores nombrados para la dirección de las manipulaciones en los remedios químicos, realizando Vito Cataldo "relaciones para los remedios químicos que había de obrar". A.G.P., Libro de registro $n^{\circ} 557$.

11 A.G.P., Sección Administrativa. Leg. 429. En VAlVERDE, J.L., y SÁNCHEZ TÉLlEZ, M.C. (1977), "El laboratorio Químico de la Real Botica, (1693-1700)". Ars Pharmaceútica, tomo XVIII, núm. 1.

En el año 1700, una resolución real comunicada al sumiller de corps, de 11 de julio, exigía que, tanto el espargírico mayor como el boticario mayor, debían seguir lo que se mandaba en la Etiqueta para la presentación de cuentas. Esta comunicación fue consecuencia de una duda planteada respecto de la intervención del veedor y contador en la toma de cuentas. A.G.P., Libro de Registro $n^{\circ}$ 557, folio 110/87.

12 Podemos hallar descritos los utensilios y el material preciso para la elaboración de composiciones en el Laboratorio de la Química como retortas, campanas, matraces, bochos, pelícanos, sacholas, embudos, canales, almireces, orinales, etc, en SÁNCHEZ TÉLLEZ, M.C. (1977). Estudio histórico de la Botica de Palacio como institución Real. Tesis doctoral inédita. D.H.F. y L.F., Facultad de Farmacia, Granada, p. 560.

13 Para mayor información sobre las elaboraciones que contiene este relación remitimos a GARCíA DE YÉBENES TORRES, P. (1984), pp. 218-221. 


\section{LA INTRODUCCIÓN DE LA PRÁCTICA QUÍMICA EN LA REAL BOTICA ESPAÑOLA}

hierro, estaño, cinabrio, oro, plata, etc.), recogiendo también remedios galénicos (triaca, todo tipo de hierbas, etc.) que, sin duda, pretendían enriquecer su alcance terapéutico. Los métodos de elaboración, basados principalmente en el tratamiento con calor en las más variadas de sus aplicaciones, nos son confirmados por la importancia de los hornos pedidos para las instalaciones del laboratorio.

Los productos que Vito Cataldo propuso comenzar a elaborar, analizados con el fin de vislumbrar hasta qué punto fue innovador en su práctica química, nos llevan a concluir que este boticario espargírico napolitano fue un experto en las prácticas de la Chimia, es decir en las manipulaciones químicas inherentes a los procesos de destilación, extracción, sublimación, calcinación, etc., por medio de las cuales se proponía la elaboración de nuevos medicamentos, de origen químico principalmente, y de administración externa e interna al paciente. Con una búsqueda en textos para la elaboración de medicamentos contemporaneos a estos acontecimientos, hemos hallado que la práctica totalidad de los medicamentos que Cataldo presentó en la relación propuesta para comenzar sus elaboraciones se corresponden con las elaboraciones comunes en textos de la práctica químico-espargírica, y que en las farmacopeas oficiales hasta entonces editadas no se incluyen prácticamente ninguno de los fármacos relacionados.

Tenemos constancia del trabajo realizado por estos tres expertos, pero siempre en documentos afines a la Real Botica; asímismo conocemos las dotaciones económicas con las que la Casa Real se comprometió a recompensar la labor de estos individuos. Sigue siendo una incógnita para nosotros saber si realmente este Oficio de la Química fue autónomo, pues todo apunta a pensar que el boticario mayor, Juan de Moya Salazar (1689-1701), fue quien siempre canalizó sus solicitudes y entregas de asignaciones económicas ${ }^{14}$. La Real Cámara jamás libró ninguna cantidad de dinero a

\footnotetext{
14 En el año 1700, se quejaba Juan del Bayle -espargírico mayor- al sumiller de corps, que teniendo una consignación de 1.000 Doblones (desconocemos si como consignación total del empleo de la Química o como consignación personal), lo empleaba casi todo en medicinas químicas, pidiendo por ello se le abonaran 700 escudos que, situados en los caudales de la Bailía General de Aragón, habían quedado "vacos", por muerte de quien los había tenido asignados; con fecha 8 de marzo de 1700 se comunicaba este extremo al Conde de Benavente. A.G.P., Sec. Adm., leg 429. 1700-1709.

Esta cantidad aparece en otros documentos como 1.000 Ducados. Además el 11 de julio de 1700 se disponía pagar una cantidad que ascendía a $4.353 \mathrm{Rv}$. para gastos de la Química, disponiendo se pagaran también los 1.000 Ducados mencionados. Esta es una resolución real comunicada al sumiller de corps como consecuencia de un memorial elevado por Juan del Bayle. A.G.P., Libro de registro $n^{\circ}$ 557. folio 110/87.

Quién asumía realmente el gasto de los medicamentos químicos que el Rey y su familia consumían, nos es desconocido. En teoría debía ser el presupuesto asignado al Oficio de la Química y el espargírico mayor quien las presentara; pero cuando, en la primera década del siglo XVIII, se le exigieron al boticario mayor la presentación de las cuentas de la Botica de estos años, éste se excusó de presentarlas con argumentos varios, y entre ellos que "los gastos de las medicinas Chymicas que oy son de gran costa".
} 


\section{PILAR GARCÍA DE YÉBENES TORRES Y M. ${ }^{a}$ LUISA DE ANDRÉS TURRIÓN}

este Laboratorio, excusándose por "hallarse mal de fondos", y desvió el pago de estos caudales al llamado "apartado de gastos secretos de S.M."15.

El Real Oficio de la Química tampoco conseguirá tener nunca una ubicación específica en aposentos reales. A comienzos de 1695 se iniciarían los trámites para montar dos habitaciones en el patio de la Real Botica, en el edificio de la Casa del Tesoro, para el recién creado Laboratorio; una para la oficina de hornos y hornillos y otra para la reposición de los medicamentos químicos ${ }^{16}$. De nuevo, por falta de fondos, el coste presupuestado para la realización de estas obras de acondicionamiento no fue librado.

La Real Botica compartió con estos expertos químicos instalaciones y presupuesto hasta sus renuncias y ceses en los cargos. Vito Cataldo, el mejor químico que hubiera en el reino de Nápoles (así se le conoció en España) ${ }^{17}$ pedirá licencia para regresar a su tierra, a finales de 1697, dadas las condiciones tan desfavorables en que tenía que trabajar. Allí se establecería con botica abierta, siempre adornada con las

Cuentas fenecidas de la Real Botica. Memorial de Luis Riqueur al conde de Benavente, sumiller de corps, en 1708. A.G.P., Felipe V. Cámara, leg. 325.

15. Memorial de Juan de Moya de 16 de enero de 1694. En este años existen diferentes informes del espargírico mayor, Vito Cataldo. A.G.P., Sec. Adm., leg. 429.

Juan de Moya Salazar, boticario mayor, en memorial al sumiller de corps, suplicaba que se hicieran efectivos estos caudales, el 8 de mayo de 1694. A.G.P., Libro de Registro ${ }^{\circ}$ 557. folio $57 / 35$.

El 18 de julio de 1698 Bayle indicaba que él mismo había suplido esta cantidad para poder continuar con las elaboraciones. A.G.P., Libro de Registro $n^{\circ}$ 557, folio 91/68.

Felipe de Torres y Salazar, del Consejo de S.M., a 29 de julio de 1700, habla del dinero entregado a Juan del Bayle para gastos del Oficio de la Química. Existen memorias presentadas por Juan del Bayle sobre diferentes gastos, así como certificaciones de Manuel Henríquez de Zeaorrote de la aprobación de diferentes cuentas presentadas. Los caudales para estos gastos se pagaron por vía de gastos secretos, por hallarse mal de fondos la Real Cámara. También hay listas de compras realizadas por la Real Botica en los últimos años del siglo XVII, para consumo en el Oficio de la Química. A.G.P., Sec. Adm., Cuentas, 1687-1700, leg. 6658.

16 En Madrid, a 7 de marzo de 1695, fue consultado el Conde de Benavente para que opinara acerca de la separación de un cuarto en la Casa del Tesoro para los remedios químicos. El cuarto sería separado de la Real Botica, viéndose también afectada por la separación de una habitación para el médico y otra para hacer un lavadero en la Plazuela de Palacio. A.G.P., Libro de Registro $n^{\circ} 547$, folio 91 vto.

Según el informe del conde de Benavente de 22 de febrero de 1695 , el coste de reparar las habitaciones ascendía a $20.000 \mathrm{Rv} .$. El 19 de marzo del mismo año se daba la orden de reparar las habitaciones que no hicieran falta a la Botica. A.G.P., Sec. Adm. leg 429.

En los pliegos de cuentas de la Real Botica de finales del siglo XVII aparecen reseñados gastos ocasionados con motivo de las obras en el patio de la fuente de la Botica para separar y construir las habitaciones de la química. A.G.P., leg. 6658 . 3260.

17. Resolución sobre criados y dependientes de la Real Cámara, de 4 de marzo de 1701. A.G.P., leg. 


\section{LA INTRODUCCIÓN DE LA PRÁCTICA QUÍMICA EN LA REAL BOTICA ESPAÑOLA}

armas reales de los austrias españoles ${ }^{18}$. Sus ayudantes, aburridos y cansados, también abandonaron ${ }^{19}$.

18. El coste presupuestado para la realización de estas obras de acondicionamiento de las habitaciones para la Química (20.000 reales de vellón) no fue librado y Cataldo se vió obligado a montar, en su propia vivienda, el centro de operaciones químicas.

Rondaba el mes de septiembre de 1697 cuando Vito Cataldo, "manipulante de lo químico", elevaba un memorial a S.M. manifestando el desconsuelo que padecía al carecer de medios con los que traer a la Corte de Madrid a su esposa e hijos. El sumiller de corps, el 25 de septiembre de 1697 elevaba el memorial a S.M., aceptando el Rey dar los medios suficientes. A.G.P., Libro de Registro $n^{\circ}$ 557. Sumillería de Corps. 1687-1706. Folio 81159 .

El 2 de noviembre de 1697 le fueron entregados 88 doblones en cumplimiento de los cien que le fueron concedidos.Vito Cataldo se quejará de la mala acogida, por parte de los médicos y boticarios españoles, a sus ideas y a su persona.

Pese a la colaboración impuesta desde sus superiores administrativos al boticario mayor, Juan de Moya y Salazar, para que el Oficio de la Química progresara (Colaboración voluntaria y sin objeciones según otras investigaciones. VALVERDE, J.L., y SÁNCHEZ TÉLLEZ, M.C., (1977)), la innovación que trajo consigo Cataldo no agradó ni a médicos ni a boticarios, apareciendo toda clase de inconvenientes y dificultades en su trabajo.

Vito Cataldo dejó de ser químico de Carlos II, regresando a Nápoles donde se estableció con botica abierta. Por Real Cédula de 8 de junio de 1698 se le concedió permiso para poner las armas reales en su botica, librándole de la inspección del Protomedicato que le correspondiera. Cuando falleció el último de los Austrias, Cataldo se vió en la necesidad de suplicar le fueran prorrogadas estas gracias, aceptando la prorroga el protomédico más antiguo del Tribunal Napolitano, Lucas Tozi.

El sumiller de corps, el Conde de Benavente, se hacía eco de estas pretensiones y solicitaba los informes pertinentes al Protomedicato de Nápoles, a 27 de mayo de 1702. A.G.P., Felipe V. Cámara. Leg. 3260.

La confirmación de poder poner el escudo y reales armas en su botica, y la exención de la visita de los ocho del colegio, es registrada el 27 de mayo de 1702. A.G.P., Libro de Registro ${ }^{\circ}$ 557, folio $124 / 101$ vto.

19. La situación económica de los componentes del oficio de la Química tampoco fue desahogada. El Rey, en el año 1697, concedió licencia a los ayudas de la Química para que volvieran a sus casas, y para poder llevar a cabo esta salida se les tuvo que socorrer con una ayuda de costa de cincuenta Doblones a cada uno. Según la comunicación del sumiller de corps, el Conde de Benavente, de 18 de mayo de 1697. A.G.P., libro de registro $n^{\circ}$ 557. Extractos de resoluciones y consultas; Sumilería de Corps, 1687-1706; folio $77 / 54$ vto.

Juan Bautista Pizzi y Nicolás Crezenzo, los dos ayudas de la Química ya mencionados, regresaron a Nápoles y solicitaron que, una vez en Nápoles, les fueran concedidos de por vida los cuatro reales de plata al día que cobraban de goce en la Corte. Solicitaban también que fueran exentos de justicia ordinaria, sujetándose al auditor general. S.M. resolvió se preparara la cantidad para pagarles, pero desconocemos la resolución a la petición de tratamiento por los tribunales. A.G.P., Libro de Registro $n^{\circ} 557.15$ de junio de 1697, folio $78 / 55$. 


\section{PILAR GARCÍA DE YÉBENES TORRES Y M. ${ }^{a}$ LUISA DE ANDRÉS TURRIÓN}

\section{2.b. Juan del Bayle, espargírico mayor de S.M.(1699-1707).}

Tras la marcha de Cataldo, tomará las riendas del Real Oficio de la Química Juan del Bayle, boticario de origen aragonés ${ }^{20}$, con unos inmejorables informes tanto de índole personal como científica y fuertemente apoyado por relevantes individuos, miembros todos ellos de la Corte madrileña como los doctores Rivas y Camps, el marqués de Valdeolmos y el propio sumiller de corps, conde de Benavente.

Fue examinado y aprobado por el Tribunal del Protomedicato en 1674, lo que le daba opción para poder ejercer como boticario en cualquier localidad dentro del territorio español.

En 1697, tras superar el examen al que de nuevo le sometería el Protomedicato, ahora por pretender utilizar su profesión al servicio directo de la corona, se le nombra como "espargírico mayor, ensayador de metales, boticario galénico y espargíri$c^{\prime \prime 21}$; conseguirá pertenecer al elenco de servidores del monarca Carlos II, ocupando el doble puesto de ayuda supernumerario de la Real Botica y colaborador o sustituto de Vito Cataldo en el trabajo de la química ${ }^{22}$. Su nombramiento causaría un tremen-

20. Del pueblo de la Almunia de Doña Godina. En 1708 dejó la Corte para ir a recuperar su salud a esta localidad, refiriéndose a su tierra.

Tambien se menciona su lugar de origen en informes a cerca de su valía profesional, cuando se abrió la posibilidad de entrar en el Laboratorio de la Química y en la Real Botica, no habiendo más precisiones biográficas.

A.G.P. Exp. Personales. Caja $91 / 6$.

21. A.G.P., Exp. Personales. Caja $91 / 6$.

Algo más tarde, tras superar la correspondiente prueba, ya en Madrid, este mismo Tribunal le nombrará "boticario racional y espargírico, teórico y práctico", juzgándole no sólo apto, sino con capacidad de "crear escuela aparte". A.G.P., Sec. Adm., Leg. 429. En VegA PORTILla, J., (1946).

22. El 2 de octubre de 1697 juró una plaza "supernumeraria" de ayuda de la Real Botica, la que compatibilizaba con el trabajo de químico, al lado de Vito Cataldo, con las ausencias, enfermedades y futura de la plaza de espargírico mayor, que tenía Cataldo. Juramento tomado por Juan de Córdoba Cárdenas, en manos del Conde de Benavente, sumiller de corps, el 2 de octubre de 1697, según la fórmula siguiente:

"Juan del Baile

Jurais a Dios Trino, y uno, de servir bien y fielmente al Rey nro. Sor. en la plaza supernumeraria de ayuda de su Rl. Botica, con las ausencias y enfermedades y futura de la plaza que hoy tiene Victor Cathaldo, de manipulante de lo químico

R. Si juro

Si asi lo hiciereis Dios os ayude, y si no os lo demande

R. Amen."

A.G.P. Exp. Personales. Caja 91/6.

Fue confirmado en el nombramiento de ayuda supernumerario de la Real Botica por Real Orden de 29 de octubre de 1698.

Como casi todos los empleados de la Familia Real sufrió retraso en el cobro de sus salarios. Según sus propios testimonios, andaba endeudado, y el 24 de noviembre de 1698 solicitaba se le pagara los 8 


\section{LA INTRODUCCIÓN DE LA PRÁCTICA QUÍMICA EN LA REAL BOTICA ESPAÑOLA}

do desagrado en determinados ambientes palaciegos para nada deseosos de relacionar a la Real Botica con el trabajo de los químicos 23 , ascendiendo a la plaza de espargírico mayor el 1 de enero de 1699. Gran influencia en la concesión de este empleo tuvo la excelente intervención de Bayle como respuesta a unas consultas que le formulara el célebre médico de la universidad de Montpellier, Raymond Vieussens; este facultativo enviará informes a los médicos de la Cámara de Castilla propiciando su nombramiento ${ }^{24}$.

meses que llevaba trabajando; como respuesta obtuvo la libranza de medio año de gajes (46.875 Mrv.). A.G.P., Exp. Personales. Caja 9116.

A partir del día 9 de febrero de 1700, se mandó situar el sueldo de Bayle, que ascendía a setecientos escudos de plata, en la nómina de la Bailía General de Aragón, sustituyendo los 1.000 Ducados que tenía señalados en la Real Cámara. Certificación de este asunto extendida por Joseph de la Peña, secretario de S.M. y oficial mayor de la Protonotaria del Reino de Aragón. A.G.P. Exp. Personales. Caja 91/6.

Desconocemos la razón por la cual Bayle solicitaba que el salario se le localizara en estos caudales, a no ser pensando en un futuro retiro a aquellas tierras. El espargírico mayor hacía esta representación con fecha 8 de marzo de 1700, solicitándolo por haber quedado una vacante al morir un tal Miguel Matheo. A.G.P. Libro de registro $n^{\circ}$ 557. Sumillería de Corps.

23. Intervino el Bureo con un informe al respecto: Calificaban el hecho de que Bayle entrara en una plaza supernumeraria de ayuda como "contraórdenes y reformaciones", pues no era sabido que hubiera químicos en la Botica; ponían en tela de juicio el poder hacer asiento del empleo en los libros de la Real Casa simplemente por una certificación del secretario del sumiller de corps, y advertían que para poderle dar el goce de ayuda sería necesario que el gobernador de Hacienda aumentara el caudal en 155.162 Mrv./año, 61.412 Mrv. por la ración y 93.750 Mrv. por gajes. El 9 de agosto se dió luz verde al aumento.

24. Este facultativo envió informes a los médicos de la Cámara de Castilla propiciando su nombramiento como espargírico mayor. Se atribuye a este boticario-espargírico una obra que, probablemente, fue la contestación científica que dió al escrito llegado de Francia del médico Raymundo Vieussens (o Veusens). Resulta curioso que el mismo Bayle consultaba al Rey el título con el que había de firmar la respuesta dada; se pidió informe al Protomedicato y, privadamente, al Doctor Rivas. Así lo comunicaba, el 25 de octubre de 1699, el sumiller de corps. Pasados unos meses, Juan del Baile solicitó se le entregaran de los caudales de la Real Hacienda, 36 Doblones para imprimir esta respuesta. Comunicación del sumiller de corps a S.M. de fecha de 5 de marzo de 1699. A.G.P. Libro de Registro $n^{\circ}$ 557; folios $95 / 72$ vto y $99 / 76$ vto.

En un informe de 4 de marzo de 1701 acerca del Oficio de la Química se mencionaba tambien al Doctor Vieussens:

“.../ La plaza de espargirico mayra. es nueva y originada de que en Madrid se avían introducido algunos estrangeros a las curaciones quimicas y su Mgd. (que Ds. aya) en tiempo del Duq. del Infantado resolvio se embiare a Napoles por el mejor quimico que hubiese en aquel Reyno y aviendose encargado al Virrey hizi conducir a esta Corte a Victor Cataldo que se mantubo algunos años en ella obrando en las manipulaciones de su ciencia con grande satisfaccion y aviendosele concedido licencia pa. restituirse a su casa, Juan Bayle por su ciencia y esperiencia y pr. considerarse que en esta facultad es el unico y con singular aprovacion del Protomedicato y q. a representacion suya poniendo la o. en las Rs. manos de Su Mgd con el motivo de haber satisfecho a unos experimentos q Reymundo de Ussens medico de Monpeller embio a los de Camara de esta Corte se sirvio concederle el titulo despachado pr la Camra de Castilla de espargirico mayr de lo quimico haciendole mrd de que en lugar de los dos Rs de a ocho al dia q. gozava en la Consignacion de la R Camra, se le situasen y pagaren 700 escudos de plata doble en la Baylia g. de Aragon. Esta facultad y laboratorio esta independiente de la Rl. Botica y segun sus creditos y lo que a mi me 


\section{PILAR GARCÍA DE YÉBENES TORRES Y M. ${ }^{a}$ LUISA DE ANDRÉS TURRIÓN}

Los servicios de Juan del Bayle no se limitarían a estos dos ámbitos que físicamente se confundían en las dependencias de la Casa del Tesoro. La llegada a España de un peculiar sujeto procedente de Nápoles (1698), Roque García de la Torre, va a

ha manisfestado la experiencia tendre por del Rl servicio de VMg se le amantenga en la misma comformidad que asta aqui pues allo q. el recurso de todos los medicos pa. remedios especificos y grandes es a su oficina.I...". A.G.P., R.C., Felipe V, leg 311, (6).

La participación de Juan del Bayle en la polémica suscitada por Vieussens sobre la existencia de una sal ácida en la sangre humana, obtenida por destilación de la misma, la recoge el trabajo de GAGO,R.; OLAGÜE,G. Y CARRILLO,J.L., (1981).

El 25 de octubre de 1698, S.M. resolvió que a Juan del Bayle se le diera el renombre de espargírico mayor, expediendo Real Decreto a la Cámara de Castilla para que, en esta vía, se le diera el título, haciéndose el asiento en los libros de la Veeduría y Contaduría, perdonándole el pago de la media annata y otros gastos como consecuencia de su nombramiento.

El 12 de mayo de 1699 juró este cargo. Con efecto desde 1 de enero de 1699 le fueron concedidos dos reales de a ocho al día, en los caudales de la Real Cámara, los mismos que se asignaron a Vito Cataldo en las Rentas de Nápoles.

Extracto del nombramiento: "Don Carlos (...) por cuanto teniendo consideración a la relevante habilidad que asiste en vuestra profesión, a vos Juan del Baile, que servis de manipulante y ayuda de mi boticario mayor y al acierto que habeis respondido a un papel escrito por Reymundo Veusens primer médico de Francia a mi protomédico por decreto señalado de mi Rl. mano de 29 de oct de 1698, he tenido por bien de haceros merced (...) de el titulo de espargírico mayor. $Y$ en su conformidad mando se os reciba el juramento (...)".

El estado de la Real Hacienda era malo y el nombramiento se produjo sin señalar el sueldo que correspondería al título. En un principio, aunque desconocemos la fecha exacta, se le asignaron dos reales de a ocho al día que gozaba su antecesor por la Real Cámara. Más adelante, se le situaron 700 escudos de plata doble en la Bailía General de Aragón.

Habiendo pasado su nombramiento de espargírico mayor por la Real Cámara de Castilla con todos los honores, goces y emolumentos, y considerándose el propio Bayle de "mas grado que el boticario mayor", pedía una ayuda de costa de jornada, la ayuda de costa de San Juan y Navidad, o un puesto correspondiente al boticario mayor en fiestas. De primera contestación le respondieron que no estaba en planta (de la Real Casa), y rápidamente solicitó se le considerara como al boticario mayor. Informe dirigido al conde de Benavente, sumiller de corps, exponiendo las pretensiones de Juan del Bayle. A.G.P., Sec. Adm., Leg. 429.

Se le concederá residencia en la Casa del Tesoro para que estuviera siempre cerca del laboratorio. El conde de Benavente, el 19 de febrero de 1698, reflejaba la solicitud del cuarto, que había quedado vacio al morir su anterior morador Francisco Barrero, ayuda de la furriera y tapicería de la Reina. A.G.P., Libro de registro $n^{\circ} 557$, folio $86 / 63$ vto.

Como al resto de empleados significados de S.M., se le concedió el 21 de junio de 1700 un balcón en la Plaza Mayor de Madrid, en las fiestas de toros. Cada persona tenía asignado un sitio en estos balcones. Así aparece en el grabado "Plaza Mayor de Madrid, con el plan de distribución del público asistente a una fiesta real de toros". Anónimo, primer tercio del siglo XIX. Diseño a pluma y aguados en tinta china. Fachadas Norte, Oeste, Sur, Este. MUSEO MUNICIPAL DE MADRID.

Esta prerrogativa igualaba en cierto modo el cargo de espargírico mayor al de boticario mayor, habiendo solicitado Bayle este balcón de repartimento. Solicitó ésto mediante memorial. La comunicación de la resolución se hacia en 20 de junio de 1700. A.G.P., Libro de Registro n ${ }^{\circ}$ 557. Sumillería de Corps. 


\title{
LA INTRODUCCIÓN DE LA PRÁCTICA QUÍMICA EN LA REAL BOTICA ESPAÑOLA
}

vincularle con las tareas que éste realizará en otro laboratorio alejado del palacio real (en la calle de Leganitos de Madrid), en el que se llevaron a cabo experiencias vinculadas a prácticas alquimistas. De nuevo, varios personajes salen a escena: el conde de Benavente, sumiller de corps y el propio Juan del Bayle. El Real Tribunal del Protomedicato se desentendió del tema pues esta labor no se realizaría dentro del palacio real y además porque el citado García de la Torre no pertenecía a ninguna de las profesiones que estaban bajo su jurisdicción ${ }^{25}$.

Juan del Bayle se hará responsable de la supervisión de estas "experiencias", sin duda relacionadas con la deteriorada salud del monarca Carlos II, y de sufragar sus gastos con las asignaciones que, del apartado de gastos secretos de S.M., personalmente le entregaría el veedor y contador real. Un año durará esta situación. Al finalizar 1699, Roque García de la Torre será invitado a retirarse y Juan del Bayle proseguirá sus tareas oficiales, que debieron ser abundantes pues se le concederá la dotación de una nueva plaza de ayuda para el Laboratorio de la Química.

Tras el fallecimiento de Carlos II (1-11-1700) su figura caerá en desgracia. El comienzo del reinado de Felipe $\mathrm{V}$ y la llegada de los nuevos facultativos sanitarios que le acompañaron arruinaron sus expectativas profesionales. Duras críticas fueron llegando contra su proceder con supuestas derivaciones alquímicas, advirtiendo de la necesidad de tomar precauciones:

\footnotetext{
“... no conviene que una leve agua de chicorias manipulada por su mano la tome S.M., la Reina ni el Príncipe..."26.
}

\begin{abstract}
25. Desconocemos la dependencia administrativa que tuvo este emplazamiento con la Casa Real. Entre las personas que trabajaron en la calle Leganitos, "frente a la Alcantarilla", en el año 1699, son nombradas Roque García de la Torre y Juan del Bayle (como supervisor del trabajo y ya espargírico mayor), que llevaron a cabo investigaciones innovadoras como ensayos sobre "la piedra" y ensayos de algún medicamento secreto, que pudiera sanar al maltrecho Carlos II. Aparecen estas referencias al laboratorio de la calle Leganitos, que remitían a las cuentas del espargírico mayor en los años 1699 y 1700, para conocer su trabajo auténtico, y a los informes de Juan del Bayle. A.G.P., Sec. Adm., Leg 429.

Trabajos de investigación llevados a cabo por Mar Rey Bueno han sacado a la luz las experiencias vinculadas a prácticas alquimistas que en este laboratorio de Leganitos tuvieron lugar. REY BUENO, M.M. (1994), Consideraciones sobre un manuscrito alquímico de la Real Botica, Tesina inédita, Madrid, D.F. y T.F., Facultad de Farmacia, U.C.M..

26. Las envidias e intrigas personales, las acusaciones vertidas sobre su posible contacto con "enemigos" del bando carlista-austríaco, hicieron que su situación laboral cayera en desgracia. En el año 1708 , tal vez aprovechando que con licencia real se retira a la Almunia a curarse, se recibieron en Madrid cartas, como la de Miguel Contin y Aguirre, advirtiendo la necesidad de tomar precauciones contra el proceder profesional del espargírico. A.G.P., Exp. Personales. Caja $91 / 6$.
\end{abstract}




\section{PILAR GARCÍA DE YÉBENES TORRES Y M. ${ }^{a}$ LUISA DE ANDRÉS TURRIÓN}

Por Resolución Real (22-5-1701), Bayle fue cesado de empleo y sueldo ${ }^{27}$. Sus súplicas y reclamaciones y el apoyo de los amigos que aún le quedaban consiguieron mantenerle en la corte con el mismo salario, a pesar de que el Oficio de la Química fue inutilizado ${ }^{28}$. Definitivamente excluido de la plantilla de criados de la Real Cámara con la Nueva Planta de 1707, quedará, no obstante, obligado a residir en Madrid y a permanecer bajo real servicio "para lo que se pudiera ofrecer" 29 .

\section{2.c. Reformas borbónicas: "Unión de la Chymica a la Botica Galénica" (1707).}

La llegada de Felipe $\mathrm{V}$ a España fue fundamental en la particular historia de su Real Botica. El monarca vino acompañado por un equipo de profesionales sanitarios que debieron, cuando menos, asombrarse del funcionamiento de aquella Oficina; no existían en ella libros actualizados para la consulta en el trabajo de los boticarios; las prácticas "químicas", la elaboración de medicamentos mediante manipulaciones químicas y la introducción efectiva de medicamentos químicos asi elaborados, se realizaban separadamente de las "galénicas" por unos individuos de dudosa reputa-

27. "Excusar a Juan del Bayle", suprimiéndole los 700 escudos que finalmente le fueron concedidos en la Bailía General de Aragón, revirtiendo este dinero en beneficio de la Real Hacienda. Resoluciones sobre criados de la Real Cámara. A.G.P., Libro de Registro $n^{\circ}$ 557. Sumillería de Corps. 1687-1706.

Por resolución real de 22 de mayo de 1701 Bayle quedó excusado, según la comunicación de fecha 20 de junio del mismo año de Martín Henriquez de Ceaorrote, veedor general. A.G.P., Leg. 3260.

Él trató de revocar la decisión por medio del envío de memoriales y en compensación a sus méritos: sin embargo, se confirmó la decisión en diferentes ocasiones. Sólo el 31 de agosto de 1701, S.M. consentía en dar a Bayle una "sobre carta". S.M. mandaba se observara lo resuelto en este tema, comunicándolo el sumiller de corps en respuesta al memorial de Juan del Bayle, en 20 de junio de 1701; otra resolución en el mismo sentido se comunicó el 14 de julio de 1701. A.G.P., Libro de Registro ${ }^{\circ} 557$.

28. Ante las súplicas de ser nombrado nuevamente, en atención a su experiencia y ciencia en la Química, así como la aprobación especial que había tenido del Protomedicato, el 20 de junio de 1701 se resolvía continuar con sus servicios por ser el único que había en "estos dominios" y haber sido solicitado por otros reinos. Esta decisión quedará sin aplicación real y efectiva hasta años después, aunque Bayle continuará en la corte.

El 3 de diciembre de 1704 Juan del Bayle fue rehabilitado por S.M. como espargírico mayor, con el mismo goce que tenía de 1.000 Ducados, situados en la consignación de la Cámara. El 8 de enero de 1705 juró la plaza. A.G.P., Leg. 320. Felipe V. Cámara.

29. A lo largo del tiempo en el que fue espargírico mayor, Bayle solicitó varias licencias para regresar a su tierra, Aragón, en distintas ocasiones. A.G.P., Leg. 320. Felipe V. Cámara.// A.G.P., Leg. 339. Felipe V. Cámara.// A.G.P., Libro de Registro $n^{\circ} 4501$. Sumillería de Corps. Extractos de consultas y resoluciones, 1707-1745. Folio 56 vto. 


\section{LA INTRODUCCIÓN DE LA PRÁCTICA QUÍMICA EN LA REAL BOTICA ESPAÑOLA}

ción científica ${ }^{30}$. Luis Riqueur ocupará inmediatamente la plaza de boticario mayor de S.M. (1701-1737) sustituyendo a Juan de Moya que será jubilado ${ }^{31}$.

Felipe $\mathrm{V}$ recibirá con agrado la solicitud de protección de los miembros fundadores de la Regia Sociedad de Medicina y otras ciencias de Sevilla, claro exponente del progresismo intelectual español, cuyas primeras Ordenanzas habían sido aprobadas por Carlos II en $1703^{32}$. Entre las normas que éstas señalaban como de obligado cumplimiento para sus miembros se encontraba la utilización de medicamentos químicos. Buen número de individuos, vinculados al ámbito sanitario del monarca, formarán parte como primeros socios: su primer médico de Cámara y presidente del Real Tribunal del Protomedicato, Honorato Michelet; su primer cirujano, Juan Bautista Legendre; su boticario mayor Luis Riqueur y muchos médicos de su Real Cámara. Por tanto, no puede entenderse abandonada la terapéutica química por parte de Riqueur cuando éste se "olvida" del Real Oficio de la Química. Muy en contra, sus pretensiones son de unificación y fusión entre la antigua práctica galénica y la espargírica o química, en el seno de la Real Botica a su cargo.

La guerra de Sucesión, con contínuos traslados del monarca y por tanto de su boticario mayor, que siempre le acompañó, no permitieron realizar reformas en la Botica Real inmediatamente. En 1707, con el decreto de Nueva Planta se reajustará la plantilla de toda la Casa Real y será el momento aprovechado por Riqueur para

30. Tanto Juan de Moya y Salazar como Juan del Bayle, boticario mayor y espargírico mayor respectivamente, y boticarios al servicio del rey en la Real Botica, administraron y canalizaron las asignaciones económicas, que siempre provinieron del apartado de "gastos secretos de S.M.", para realizar las prácticas químicas.

31. De Luis Riqueur así como de la evolución de la Real Botica durante la primera mitad del siglo XVIII y su relación con la evolución de las instituciones sanitarias y científicas, existe un estudio detallado en GARCÍA DE YÉBENES TORRES, P. (1994).

32. En Sevilla, cristalizó en 1698 una academia médica de lineas modernas. Su primer nombre fue "Veneranda tertulia hispalense, Medica-chimica, Anathomica, y Mathematica".

En la fundación de la Regia Sociedad Sevillana, se agregaron los médicos de altos cargos de la Corte, como el médico del Cardenal Portocarrero, sanitarios cercanos a Doña Mariana de Austria, o Juan de Cabriada. Fueron aprobadas las constituciones el 25 de mayo de 1700, por su Majestad Católica, con el nombre de Sociedad Regia Filosófica y Médica de Sevilla, y después Regia Sociedad de Medicina y demás ciencias, de Sevilla. En sus primeras ordenanzas se mandaba a los socios "de habilitar en todo lo que producen los fármacos en la doctrina espagírica". Carlos II aprobó sus ordenanzas el mismo día que sus constituciones, el 25 de mayo de 1700. Felipe V, por Real Cédula de 1 de octubre de 1701, mandó protegerla. En el siglo XVIII esta institución mantuvo vivo el interés por esta ciencia, y al final del segundo decenio se nombró a un socio espargírico con la obligación de hacer experimentos químicos. Hermosilla Molina, A., (1970) Cien años de medicina Sevillana. La Regia Sociedad de Medicina y demás ciencias, de Sevilla, en el siglo XVIII, Sevilla, CSIC y otros. 
nombrar a un mozo de su botica, colaborador y amigo personal, Francisco García de Vicuña, como ayuda de espargírico mayor ${ }^{33}$.

\section{2.d. Francisco García de Vicuña, espargírico mayor de S.M. (1719-1723).}

El cargo de espargírico mayor debió convertirse en una obsesión para Riqueur que, en 1701, se autodenominará como tal aunque estaba lejos de producirse esa situación ${ }^{34}$. El boticario mayor conseguirá retirar a Bayle de su actividad en el Oficio de la Química pero no eliminar ni su plaza ni su salario, que fueron variando de localización según las reformas que la Real Casa y Hacienda estimaron necesarias. Tantos cambios llegarían a provocar gran confusión en estos ámbitos; el sueldo de espargírico mayor "reformado" será pagado con fondos de la Tesorería Mayor de la Guerra ignorando, los propios jefes de la Casa Real, cuál era la ubicación laboral de este criado de S.M. ¿estaba jubilado o reformado...? $?^{35}$.

Mientras esto sucedía, el trabajo de la química lo realizaba Francisco García de Vicuña, natural de Vitoria (Álava) que había comenzado a trabajar como mozo de oficio en la Real Botica en $1695^{36}$. Sus relaciones con Luis Riqueur y con miembros

\footnotetext{
33. Del nombramiento se dió aviso al contralor el 6 de julio del mismo año. Sorprendentemente surgió el problema de no estar comprendida esta plaza en la nueva planta, y por tanto el sueldo de 155.162 Mrv. tampoco estaba consignado en la Casa. Esta plaza no era de nueva creación, habiéndola ocupado Juan del Bayle anteriormente. A.G.P., Exp. Personales. Caja $423 / 15$.

Y por razón que desconocemos (a parte de las económicas) se había prescindido de ella. Después de escritos y súplicas se le asignó de nuevo el caudal a la plaza de ayuda de espargírico, aumentando el mayordomo mayor la consignación de la Casa, dando el aviso al maestro de la Cámara el 1 de septiembre de 1707. Una vez solventados estos problemas debía hacer frente al pago de la media annata; sin embargo pidió que se le descontara el dinero de lo que le debían de los salarios como mozo de oficio.

34. En la lista de socios de la "Veneranda" aparecía Luis Riqueur con el cargo de espargírico. HeRmosilla MOLINA, A. (1970), p. 723.

35. Abundante documentación que refleja toda esta confusión aparece en GARCíA DE YÉBENES TORRES, P. (1994), pp. 215-232.

36. El 24 de octubre de 1695 juró la plaza de mozo de oficio. Certificación hecha con motivo del juramento, al promocionar a ayuda a Francisco Arias. A.G.P., Exp. Personales. Caja 30/31.

Pese a la incompatibilidad por el cargo que ocupaba en la Real Botica, tuvo botica propia en Madrid, en la plaza de Santo Domingo. Otorgó carta de dote el 14 de julio de 1699 y casado con Manuela de Ano (que aportó al matrimonio una dote de 56.424 Rv.), es gracias a este matrimonio por lo que llegaría a ser propietario de una botica en Madrid, en la plaza de Santo Domingo; esta botica perteneció a Antonio de Ano, padre de su esposa, a cuya muerte la heredó Francisco de Ano, hermano de la esposa, y ésta la heredó a su muerte. Es una gran excepción, ya que un boticario al real servicio no podía tener botica propia. Por esta incompatibilidad como administrador de la botica actuó el boticario Baltasar Gómez de Burón. Esta botica de Santo Domingo tuvo una distinguida clientela, como refleja el documento de partición de bienes de $G^{a}$. Vicuña: Marqués de la Olmeda, de Alcañices, de Navamorquende, de Leganés,
} 


\section{LA INTRODUCCIÓN DE LA PRÁCTICA QUÍMICA EN LA REAL BOTICA ESPAÑOLA}

de su familia fueron siempre excelentes ${ }^{37}$, depositando el boticario mayor en él toda su confianza en la toma de importantes decisiones de índole económica y administrativa del Oficio de la Real Botica.

Cuando García de Vicuña llevaba más de doce años como ayuda de espargírico mayor, morirá Juan del Bayle, pasando a ocupar el cargo (1719) ${ }^{38}$ y quedando bajo las órdenes de Riqueur hasta su fallecimiento, cuatro años más tarde ${ }^{39}$. Se sentenció de esta forma el final para el Oficio de la Química; el puesto de espargírico mayor se unió junto con el de destilador mayor a la plaza de boticario mayor de S.M..

3. EXTINCIÓN DEL REAL OFICIO DE LA QUíMICA (R.D. 7-7-1723). LUIS RIQUEUR, BOTICARIO MAYOR, ESPARGÍRICO MAYOR Y DESTILADOR MAYOR DE S.M. FELIPE V.

Al igual que sucediera dos años antes (Resolución 8-3-1721) con el Real Oficio de la Destilación, cuya función esencial había sido la obtención de aguas destiladas y de aceites medicinales, en 1723, debido a la muerte del hasta entonces espargírico mayor, Francisco García de Vicuña, se decidió que este cargo se uniera al de boticario mayor, así como el Oficio de la Química al de la Botica ${ }^{40}$.

de la Vega de Santa María, de Castelar, y Conde de Fuensalida, de Liria y de las Torres. DE ANDRÉS, F., (1984) Farmacia y Farmacéuticos a través del Protocolo Notarial de Madrid (segundo cuarto del siglo XVIII), Tesis Doctoral inédita, Facultad de Farmacia U.C.M., Madrid, p. 328.

37. Intervino como apoderado en diferentes negocios de Jacobo Riqueur, como la fábrica de pan y su distribución para el Ejército.

38. El 22 de febrero de 1719 solicitó García de Vicuña se le concediera la plaza, con el goce que Bayle tenía pero a cobrar por la Maestría de Cámara y no por la Tesorería de la Guerra, como hasta entonces estaba fijado. Y así se lo concedieron. El 24 de febrero de 1719 fue nombrado, haciéndose el asiento correspondiente en los libros del grefier y tomar razón en la Tesorería General de S.M..

39. Falleció en su casa de la calle de la Puebla (de Madrid), siendo enterrado en el convento de Nuestra Señora del Rosario, e inscrito el fallecimiento en la Iglesia de San Martín, libro ${ }^{\circ} 14$ de difuntos. Había nombrado herederos a sus tres hijos. Una de sus hijas heredó la botica entera, al estar casada con el boticario Martín de Urruzola, quién se hizo cargo de la administración. A su muerte sus bienes totales ascendían a 391.257 Rv. 19 Mrv., debiéndole la Real Hacienda salarios desde 1715 (aproximadamente $13.690 \mathrm{Rv}$ ). Formó una amplia biblioteca, de 101 volúmenes relacionados con medicina, cirugía, física, ciencias naturales, química y farmacia. ANDRÉs, F. de, (1984).

40. Resolución dirigida al Conde de Peñaranda y sumiller de corps, a 7 de julio de 1723. El 8 de julio se da informe al veedor y contador de la Cámara para que se asentaran en el libro los nombramientos. A.G.P., Leg. 3264.

Esta resolución fue comunicada de unos jefes a otros dentro de los altos cargos de la Casa Real. El Marqués de Grimaldo al Conde de Peñaranda, éste a José Rodrigo,... Y sucedía en los días del mes de junio de 1723. La plaza de espargírico mayor se reunía a la del jefe de la Botica, y los gajes del espargírico se dejaban para el ayuda más antiguo. A.G.P., Sec. Adm. leg. 690. 


\section{PILAR GARCÍA DE YÉBENES TORRES Y M. ${ }^{a}$ LUISA DE ANDRÉS TURRIÓN}

El Oficio desapareció como organismo autónomo, pero el trabajo se incorporó como rama fundamental de la elaboración de medicamentos en la Real Botica ${ }^{41}$.

Luis Riqueur había conseguido su objetivo tras más de veinte años de intentarlo; por fin era espargírico mayor, como figuraba en la lista de socios de la Regia Sociedad médica sevillana desde 1701. Durante todo este tiempo trabajaría en la remodelación de la que sería su nueva Real Botica. Compró muchos libros para esta Oficina, comenzando por todos los códigos y farmacopeas que sobre la preparación de medicamentos hubieran sido publicados en España. Completará la Librería de la Real Botica con textos relativos a la práctica química de autores como Pedro Poterio, Luís de Oviedo, Nicolás Lemery, Félix Palacios, Francisco Vélez de Arciniega, José Assín y Palacios, Gerónimo de la Fuente, Miguel Martínez de Leache... ${ }^{42}$.

El personal de la Real Botica asignado a la rama de la química, formado especialmente por Riqueur como responsable absoluto, estuvo integrado por dos ayudas y un mozo de oficio destinado en la química, sobre los que recaía el trabajo real. Bartolomé Pérez Durán, que ocuparía la plaza de ayuda de espargírico mayor más antiguo $(1723)^{43}$, segunda en el escalafón de la Real Botica, llevaba trabajando con él, desde 1711, en operaciones espargíricas. Será el sustituto de Riqueur en la dirección de esta Oficina. Francisco Corral, nombrado ayuda de espargírico mayor más moderno (1723) practicó, desde 1715, como mozo de oficio. Finalmente, José de Lama sería el mozo destinado a la Química (1723).

41. El Oficio de la Química estaba "enfermo" desde sus comienzos; el 21 de septiembre de 1694, se registra un informe sobre lo conveniente de manipular en la Real Botica los remedios químicos que estaba ya elaborando Vito Cataldo. A.G.P., Libro de Registro $n^{a}$ 557. Sumiller de corps; Extracto de consultas y resoluciones. 1687-1706. Folio 59/37.

42. Una descripción de los libros comprados por la Real Botica correspondiente a adquisiciones reflejadas en recibos de data otorgados a proveedores, así como el criterio de "biblioteca básica" para la elaboración de medicamentos transcrito en la lista de los libros que se compraron para la composición de la botica de jornada del Infante D. Felipe en su viaje a Italia en 1742, aparecen en GARCíA DE YÉBENES TORRES, P. (1994), pp. 158-160 y 345.

43. Decreto expedido por el mayordomo mayor, el Marqués de Villena. Registro de diferentes órdenes dirigidas al oficio de contralor. Estos registros, de los juramentos, se hicieron a 7 y 6 de septiembre de 1723 por José Espino y Navarro. A.G.P., leg. 3155.

Este escalafón aparece en la comunicación hecha en Valsaín al Conde de Peñaranda, el 7 de julio de 1723, acerca del extinto Oficio de la Química. A.G.P., Libro de Registro $n^{\circ} 1515$. folio 83 vto. 


\section{LA INTRODUCCIÓN DE LA PRÁCTICA QUÍMICA EN LA REAL BOTICA ESPAÑOLA}

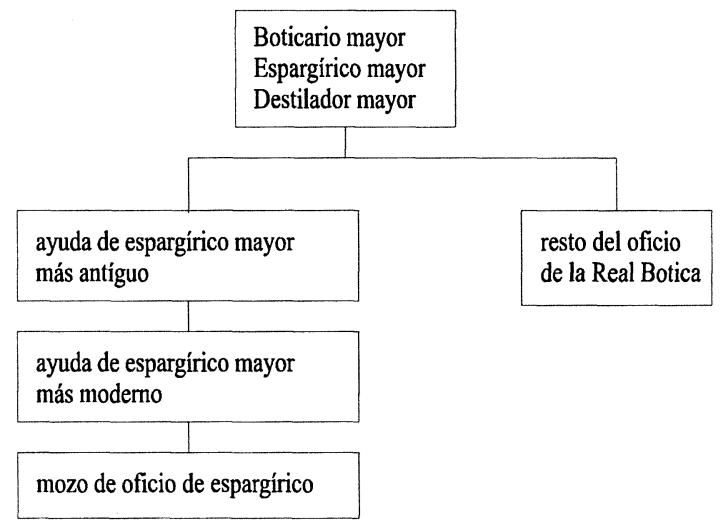

Composición de la rama del Oficio de la Química integrada en la Real Botica.

Todos los empleados que, con posterioridad, trabajaron en la Química y que diferenciaban sus empleos con el apellido de espargíricos fueron considerados como la élite dentro del Oficio Real y gozaron de un sobresueldo que premiaba su labor ${ }^{44}$.

Tras la muerte de Felipe V, Fernando VI reformó la actuación del personal de su Real Cámara mediante la Nueva Planta de 8 de abril de 174945. La "planta" correspondiente a la Real Botica incluyó:

\begin{tabular}{|c|c|c|}
\hline Boticario mayor .................................... con un salario de & 30.000 & Rv./año \\
\hline Primer ayuda & 15.000 & “ \\
\hline Tres ayudas más & 8.200 & “ cada uno \\
\hline Primer mozo de oficio & 6.000 & “ \\
\hline 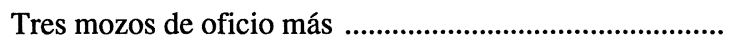 & 4.400 & “ cada uno \\
\hline
\end{tabular}

Observamos que la denominación "con destino en la Química" no se detalla, si bien la habilidad y responsabilidad continuó siendo condición necesaria para los individuos que ocuparon las primeras plazas.

44. El sueldo que se fijó a Bartolomé Pérez Durán, es decir al ayuda más antiguo de espargírico mayor, fue de $11.000 \mathrm{Rv}$. al año por los caudales de la Real Casa, salario que ya tenía fijado Francisco García de Vicuña en 1720 (como espargírico mayor). Todos los empleados que trabajaron en la Química tuvieron un sobresueldo, que premiaba el sobretrabajo y evitó "problemas".

45. A.G.P., Sec. Adm., leg. 468. 
A partir de 1753, como consecuencia de una Resolución Real, fueron incluidos en la nómina de la Real Cámara un entretenido (boticario meritorio sin sueldo) y un mozo inferior (generalmente sin estudios), destinados en los trabajos de la química.

Hasta mediados del siglo diecinueve continuarán preparándose los medicamentos químicos y realizándose todas las operaciones de naturaleza química bajo la responsabilidad del segundo boticario de la Real Botica (denominación equivalente a "primer ayuda de la Real Botica con destino en la química, primer ayuda de espargírico mayor o primer ayuda") 46 .

El Reglamento de 1798, momento de gran esplendor de la Real Botica y de la profesión farmacéutica, en el que personal farmacéutico de ésta forma parte del Protofarmacéuticato (excisión del Real Tribunal de Protomedicato con absoluta autonomía en su control profesional) señalaba, en cuanto al modo de preparar los medicamentos, que si estos eran de naturaleza galénica serían confeccionados por los boticarios de Cámara de tercera clase y si eran químicos, por el más antiguo de segunda ${ }^{47}$.

$\mathrm{Si}$ bien este reglamento no fue derogado tras la guerra de la Independencia (1808), Agustín J. Mestre (boticario mayor), en 1815, apuntó variaciones sobre aquél sin modificar los párrafos que nos interesan. El primer boticario de Cámara de segunda clase se encargaba, como en el reglamento anterior, de la elaboración de medicamentos químicos, mientras que los galénicos se preparaban por un boticario de tercera.

Tras este reglamento de 1815 , no vuelve a aparecer desglosada la preparación de medicamentos galénicos y químicos. Entre las funciones del boticario mayor se encontraría la supervisión de todos los trabajos de ensayos y análisis químicos que sus boticarios de Cámara realizaran.

\footnotetext{
46. Reglamento de 1798. A.G.P.:DOCUMENTOS DE LA REAL OFICINA DE FARMACIA, carpeta b-4-24. Reglamentos y proyectos.// 1815. Informe de A. J. Mestre al sumiller de corps., carp. $b$-4-17.

47. La plantilla de profesionales de la Real Botica se dividía en: boticario mayor, boticario de cámara de primera (que era el delegado del boticario mayor en su ausencia e individuo de su confianza), boticarios de cámara de segunda y boticarios de cámara de tercera.
} 